\title{
STABILITY ANALYSIS FOR HYBRID AUTOMATA USING CONSERVATIVE GAINS
}

\author{
Rom Langerak ${ }^{*, 1}$ Jan Willem Polderman ${ }^{*, 2}$ \\ Tomas Krilavičius *,3 \\ * EEMCS, University of Twente, P.O. box 217, \\ 7500 AE Enschede, The Netherlands, \\ $\{$ langerak,krilavic\}@cs.utwente.nl, \\ j.w.polderman@math.utwente.nl
}

\begin{abstract}
This paper presents a stability analysis approach for a class of hybrid automata. It is assumed that the dynamics in each location of the hybrid automaton is linear and asymptotically stable, and that the guards on the transitions are hyperplanes in the state space. For each pair of ingoing and outgoing transitions in a location a conservative estimate is made of the gain via a Lyapunov function for the dynamics in that location. It is shown how the choice of the Lyapunov function can be optimized to obtain the best possible estimate. The calculated conservative gains are used in defining a so-called gain automaton that forms the basis of an algorithmic criterion for the stability of the hybrid automaton. Copyright(c)2003 IFAC
\end{abstract}

Keywords: Hybrid Systems, Stability Analysis, Lyapunov Function

\section{INTRODUCTION}

The study of hybrid systems, which are systems whose behaviour can be seen as the result of the interaction between discrete and continuous dynamics, has given rise to a wealth of different models (see (Van der Schaft and Schumacher, 2000) for an overview). The hybrid automata model (Alur et al., 1993; Henzinger, 1996) is an attractive candidate as it is very general and can be seen as a straightforward extension of the timed automata model (Alur and Dill, 1994) that has been extensively studied, especially in computer science, resulting in a large body of results on (automated) analysis and design. A hybrid automaton consists of an automaton with locations and transitions (or switches) between the transitions, together with continuous dynamics in the locations (usually de-

\footnotetext{
1 Supported by the EU-IST project AMETIST

2 Supported by EU-IST program HYBRIDGE

3 Supported by the NWO project CASH
}

scribed by differential equations) and constraints on both locations and transitions.

The question of stability for hybrid automata has been known to be nontrivial. It is possible to find very simple examples (see e.g. (Branicky, 1994)) that illustrate that even if the dynamics in each location is asymptotically stable, still the global behaviour of the hybrid automaton is unstable. By now there are many results on the stability of hybrid systems (for an overview see (DeCarlo et al., 2000; Liberzon and Morse, 1999; Michel, 1999)). Many of these results address the question of the stability of a set of locations under arbitrary switching between these locations (Michel, 1999; Ye et al., 1998), for instance by checking the existence of a Lyapunov function common to all locations (Liberzon and Morse, 1999; Agrachev and Liberzon, 2001). Such a criterion would be too strong in general for hybrid automata, since there one is not interested in all possible switchings, but only in the switchings allowed by the transitions of the automaton. 
A more suitable criterion for our purposes is that of multiple Lyapunov functions (Branicky, 1994; Branicky, 1998; Liberzon and Morse, 1999). Here one assumes the existence of a Lyapunov function in each location, together with the so-called non-increasing sequence property: if a location is visited again, the value of the Lyapunov function should be less than it was at the previous time the location was entered. This is a sufficient condition for the stability of a hybrid automaton. However, the problem is that is not clear how the nonincreasing sequence can be effectively checked in general, since it would in principle require checking all possible behaviours of a hybrid system.

This problem has been tackled by constructing Lyapunov functions that are either piecewise linear (Koutsoukos and Antsaklis, 2001) or piecewise quadratic (Pettersson and Lennartson, 1996; Johansson and Rantzer, 1998; Mignone et al., 2000). In the latter case the piecewise quadratic function should be continuous on the switching boundaries, which can be checked efficiently by solving a linear matrix inequality. The approach has originally been formulated for piecewise affine systems, where the state space is divided into regions, and to each region corresponds a dynamics. It is not so easy to adapt the approach to the more general model of hybrid automata.

Therefore in this paper we take another approach that exploits the automaton structure of the hybrid automaton while taking advantage of the existence of a Lyapunov function in each location. In Section 2 we give some basic definitions about hybrid automata and stability, and define the class of hybrid automata that we study in this paper. In Section 3 we show how to calculate a conservative estimate of the gain using Lyapunov functions. It is shown how an optimal Lyapunov function can be chosen. In Section 4 we show how to use conservative gains in constructing an automaton that is used in an algorithm that detects unstable cycles in a hybrid automaton. The absence of such unstable cycles is a sufficient condition for the stability of the hybrid automaton. In Section 5 we give conclusions and some directions for future research. For basic notions of systems theory used in this paper we refer to (Polderman and Willems, 1997).

\section{HYBRID AUTOMATA AND STABILITY}

The hybrid automaton model (Alur et al., 1993; Henzinger, 1996) extends the classical notion of automaton by incorporating continuous dynamics in the locations, together with constraints at both locations and transitions.
Definition 1. A hybrid automaton is a tuple $H=$ (X, L, Init, Inv, $f, E$, Guard, Assign, $\Sigma)$ where:

- $X \subseteq \mathbb{R}^{n}$ is the continuous state space ranged over by the state vector $x$.

- $L$ is a finite set of locations.

- Init $\subseteq L \times \mathbb{R}^{n}$ is a set of initial location state pairs.

- Inv $: L \rightarrow 2^{X}$ assigns to each location $l$ an invariant to be satisfied by state $x$ while in location $l$.

- $f: L \rightarrow\left(X \rightarrow \mathbb{R}^{n}\right)$ assigns to each location $l$ a continuous vector field $f_{l}$ such that the state $x$ should satisfy $\frac{d}{d t} x=f_{l}(x)$.

- $E \subseteq L \times L$ is the set of transitions, also called switches.

- Guard : $E \rightarrow 2^{X}$ assigns to each transition a guard that has to be satisfied by state $x$ if the transition is taken.

- Assign : $E \rightarrow(X \rightarrow X)$ assigns to each transition an assignment that may alter state $x$ when the transition is taken.

- $\Sigma$ a set of transition labels. We assume a labelling function $l a b: E \rightarrow \Sigma$ and refer to transitions by their labels (assuming uniqueness).

In this paper we make a few additional assumptions:

- We assume that Init $=L^{\prime} \times \mathbb{R}^{n}$ for a set $L^{\prime} \subseteq L$ of initial locations, so that for a given initial location we can start with any state (which is technically convenient when studying stability).

- We assume that there are no invariants, i.e., Inv maps each location to the trivial condition true. This means that transitions are never forced, and it is possible to remain in a location forever.

- we assume that the dynamics in each location is linear, so $\frac{d}{d t} x=f_{l}(x)=A_{l} x, A_{l} \in \mathbb{R}^{n \times n}$.

- We assume that for each transition $e$ the guard is a hyperplane defined by an equation of the form $v_{e}^{\mathrm{T}} x=0$ for some $v_{e} \in \mathbb{R}^{n}$.

- We assume that the state is left unchanged by transitions (also called continuous switching), so for each transition $e, \operatorname{Assign}_{e}(x)=x$.

We call a hybrid automaton that satisfies these assumptions a Linear Continuous Hyperplane (LCH) hybrid automaton.

Example 2. Consider the hybrid automaton consisting of four locations, $l_{1}, \ldots, l_{4}$. The dynamics in location $l_{i}$ is given by $\frac{d}{d t} x=A_{l_{i}} x, A_{l_{i}} \in \mathbb{R}^{2 \times 2}$, $i=1, \ldots 4$. The following events can occur: $E=$ $\left\{\left(l_{1}, l_{2}\right),\left(l_{1}, l_{4}\right),\left(l_{2}, l_{1}\right),\left(l_{2}, l_{3}\right),\left(l_{3}, l_{4}\right),\left(l_{4}, l_{2}\right)\right\}$, to which correspond labels $a$ to $f$ respectively. To each event there corresponds a switching line $L_{i j}$. For instance if the automaton is in location $l_{2}$ 
there are two possible transitions: to $l_{1}$ and to $l_{3}$. The transition to $l_{1}$ is enabled if and only if $x \in L_{21}$, whereas the transition to $l_{3}$ is possible when $x \in L_{23}$.

Definition 3. A hybrid trace of an LCH hybrid automaton is a finite or infinite sequence $\sigma=x_{1} e_{1} x_{2} e_{2} \ldots x_{m-1} e_{m-1} x_{m}$, with an associated monotonically increasing timing sequence $\tau_{0} \tau_{1} \ldots \tau_{m}$ (with $\tau_{0}=0, \tau_{i} \in \mathbb{R}^{n} \cup\{\infty\}$ ), such that

- each $e_{i}$ is a transition from location $l_{i}$ to location $l_{i+1}$

- each $x_{i}$ is a mapping from $\left[\tau_{i-1}, \tau_{i}\right]$ to $\mathbb{R}^{n}$ satisfying $\frac{d}{d t} x_{i}=A_{l_{i}} x_{i}$

- initial and switching constraints and assignments are respected, so $\left(l_{1}, x_{1}(0)\right) \in$ Init, and for all $1 \leq i \leq m-1: v_{e_{i}}^{\mathrm{T}} x_{i}\left(\tau_{i}\right)=0$ and $x_{i}\left(\tau_{i}\right)=x_{i+1}\left(\tau_{i}\right)$.

Furthermore we will only consider non-Zeno traces, so we assume that if a hybrid trace is infinite, $\lim _{i \rightarrow \infty} \tau_{i}=\infty$.

Definition 4. An LCH hybrid automaton is stable iff $\forall \epsilon>0 \exists \delta>0:\left|x^{0}\right|<\delta \Rightarrow$ for all hybrid traces $x_{1} e_{1} x_{2} e_{2} \ldots$ with $x_{1}(0)=x^{0}$ and $\forall i \forall t \in$ $\left[\tau_{i-1}, \tau_{i}\right]:\left|x_{i}(t)\right|<\epsilon$. An automaton that is not stable is called unstable.

It is well known that even if for each location $l$ the dynamics is asymptotically stable, so the matrix $A_{l}$ is Hurwitz (i.e., all eigenvalues have a negative real part), still the hybrid automaton can be unstable (see e.g. (Branicky, 1994) for a simple example). We say that a hybrid automaton has Hurwitz locations if for each location $l$ the matrix $A_{l}$ is Hurwitz. Now our problem is the following: find sufficient conditions for the stability of an LCH hybrid automaton with Hurwitz locations.

\section{CONSERVATIVE ESTIMATE OF GAINS VIA LYAPUNOV FUNCTIONS}

Suppose a location $l$ is entered via a transition $a$ with a state vector $x_{a}$ and is left via a transition $b$ with a state vector $x_{b}$. An indication as to how the location contributes to the stability or instability is the ratio of the norm of the outbound state and the inbound state. A ratio below one is in favor of stability whereas a ratio above one points at instability. Of course, since the ratio depends on the actual trace and state trajectory it does not provide a feasible stability indicator. Therefore we propose to use an upperbound that only depends on the particular location and its corresponding inbound and outbound transitions instead.

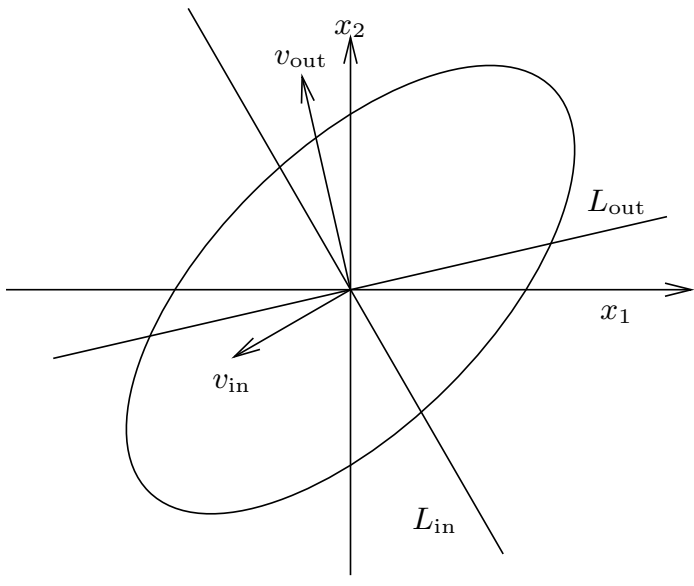

Fig. 1. The situation in a location

Definition 5. Consider an LCH hybrid automaton $H$ with Hurwitz locations. With each location $l$ we associate a symmetric positive definite matrix $P_{l}$ such that $A_{l}^{\mathrm{T}} P_{l}+P_{l} A_{l}<0$. Let $e_{\text {in }}$ represent a transition to $l$ and $e_{\text {out }}$ a transition from $l$ and let $L_{\mathrm{in}}$, given by $v_{\mathrm{in}}^{\mathrm{T}} x=0$, and $L_{\mathrm{out}}$, given by $v_{\text {out }}^{\mathrm{T}} x=0$, denote the corresponding switching hyperplanes. Define ellipsoids $E_{\text {in }}$ and $E_{\text {out }}$ as $E_{\text {in }}=\left\{x \in L_{\text {in }} \mid x^{\mathrm{T}} P_{l} x=1\right\}$ and $E_{\text {out }}=\{x \in$ $\left.L_{\text {out }} \mid x^{\mathrm{T}} P_{l} x=1\right\}$. The corresponding gain $\alpha_{\text {in } / \text { out }}$ is defined as

$$
\alpha_{\text {in } / \text { out }}=\max _{x_{\mathrm{i}} \in E_{\mathrm{in}}, x_{\mathrm{o}} \in E_{\text {out }}} \frac{x_{\mathrm{o}}^{\mathrm{T}} x_{\mathrm{o}}}{x_{\mathrm{i}}^{\mathrm{T}} x_{\mathrm{i}}}
$$

Obviously, since $V(x)=x^{\mathrm{T}} P_{l} x$ is a Lyapunov function for $\frac{d}{d t} x=A_{l} x$ we have that any trajectory that enters the location through $L_{\text {in }}$ and leaves it through $L_{\text {out }}$ has the property that the ratio of the norms of outbound and inbound states is upperbounded by $\sqrt{\alpha_{\text {in/out }}}$.

Two questions arise. Firstly, given $P_{l}$ how can we calculate $\alpha_{\text {in/out }}$ ? Secondly, it appears that the choice of $P_{l}$ affects $\alpha_{\text {in/out }}$. How to choose $P_{l}$ such that $\alpha_{\text {in/out }}$ is minimal? The latter question is treated in Section 3.2.

\subsection{Calculation of the gains}

Let us explain how the calculation for given $P_{l}$ works. For convenience we treat the two dimensional case first, see Figure 1.

The switching lines are given by $v_{\text {in }}^{\mathrm{T}} x=0$ and $v_{\text {out }}^{\mathrm{T}} x=0$ respectively. Let $\tilde{v}_{\text {in }}$ and $\tilde{v}_{\text {out }}$ be orthogonal to $v_{\text {in }}$ and $v_{\text {out }}$ respectively. Then it is not difficult to verify that

$$
\alpha_{\text {in } / \text { out }}=\frac{\tilde{v}_{\text {out }}^{\mathrm{T}} \tilde{v}_{\text {out }}}{\tilde{v}_{\text {in }}^{\mathrm{T}} \tilde{v}_{\text {in }}} \frac{\tilde{v}_{\text {in }}^{\mathrm{T}} P_{l} \tilde{v}_{\text {in }}}{\tilde{v}_{\text {out }}^{\mathrm{T}} P_{l} \tilde{v}_{\text {out }}} .
$$

If we choose $\tilde{v}_{\text {in }}$ and $\tilde{v}_{\text {out }}$ both on the same level curve, then 2 reduces to $\frac{\tilde{v}_{\text {out }}^{\mathrm{T}} \tilde{v}_{\text {out }}}{\tilde{v}_{\text {in }}^{\mathrm{T}} \tilde{v}_{\text {in }}}$. 
For dimensions $n>2$ the situation is a bit more complicated since the maximization in (2) comes into play.

First notice that

$$
\alpha_{\text {in } / \text { out }}=\frac{\max _{x_{\mathrm{o}} \in E_{\mathrm{out}}} x_{\mathrm{o}}^{\mathrm{T}} x_{\mathrm{o}}}{\min _{x_{\mathrm{i}} \in E_{\text {in }}} x_{\mathrm{i}}^{\mathrm{T}} x_{\mathrm{i}}} .
$$

Next, e.g., the numerator of (3) can easily be calculated as follows. First assume that $v_{\text {out }}$ has norm one (otherwise normalize), then determine an orthogonal matrix $V_{\text {out }}$ such that the first column of $V_{\text {out }}$ is $v_{\text {out }}$. Define $P_{\text {out }}=V_{\text {out }}^{\mathrm{T}} P_{l} V_{\text {out }}$ and $\tilde{E}_{\text {out }}=\left\{z \in \mathbb{R}^{n} \mid z_{1}=0, z^{\mathrm{T}} P_{\text {out }} z=1\right\}$. Then

$$
\max _{x \in E_{\text {out }}} x^{\mathrm{T}} x=\max _{z \in \tilde{E}_{\text {out }}} z^{\mathrm{T}} z=\frac{1}{\lambda_{\min }\left(\tilde{P}_{\text {out }}\right)},
$$

where $\tilde{P}_{\text {out }}$ is obtained from $P_{\text {out }}$ by deleting the first row and and the first column. Furthermore $\lambda_{\min }(P)$ denotes the smallest eigenvalue of matrix $P$. In a similar way the denominator of $(3)$ is obtained, resulting in

$$
\alpha_{\text {in } / \text { out }}=\frac{\lambda_{\max }\left(\tilde{P}_{\text {in }}\right)}{\lambda_{\min }\left(\tilde{P}_{\text {out }}\right)} .
$$

\subsection{Optimizing the choice of Lyapunov function}

The gains that provide a (conservative) stability indicator obviously depend on the Lyapunov functions in each location. Loosely, the fit of the level curves with respect to the trajectories determines the amount of conservatism. The better the fit, the less conservative the gain. Since Lyapunov functions are not unique, this suggests that we might be able to choose in each location a Lyapunov function that is optimal with respect to the gain. In this section we briefly explain how this can indeed be done. We confine ourselves to linear dynamics within locations and quadratic Lyapunov functions. We show that for a given Hurwitz matrix $A$ the set of quadratic Lyapunov functions is convex and compact. Furthermore the stability gain corresponding to $A$ and given switching hyperplanes depends continuously on the Lyapunov function so that at least the optimum exists. We illustrate the effectiveness of these results by means of a two dimensional example.

Let $A \in \mathbb{R}^{n \times n}$ be a Hurwitz matrix. We are interested in the set of quadratic Lyapunov functions, or, more precisely, the set of level curves corresponding to quadratic Lyapunov functions. To enable the comparison of different Lyapunov functions we choose $x_{0} \in \mathbb{R}^{n}$ and define the set

$\Omega_{x_{0}}=\left\{P \in \mathbb{R}^{n \times n} \mid A^{\mathrm{T}} P+P A \leq 0, x_{0}^{\mathrm{T}} P x_{0}=1\right\}$.

In fact, $\Omega_{x_{0}}$ is a parametrization of the level curves corresponding to quadratic Lyapunov functions and level unity.

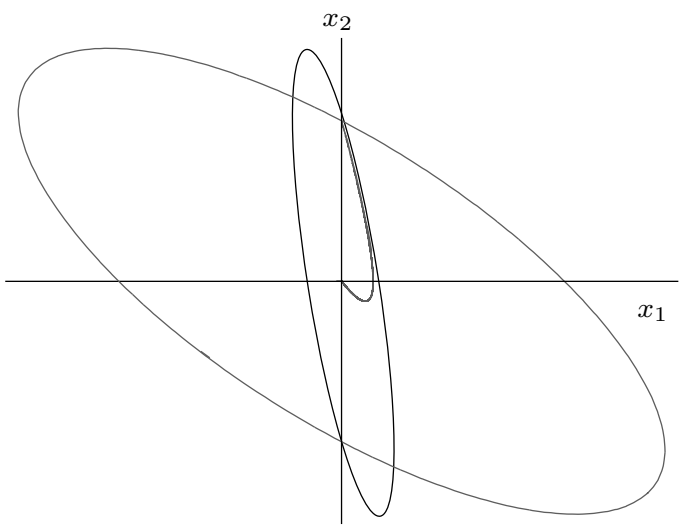

Fig. 2. Level curves of the extreme Lyapunov functions

Theorem 6.

(1) If $x_{0}$ does not belong to an $A$-invariant subspace of dimension at most $n-1$, then $\Omega_{x_{0}}$ is compact.

(2) Every $P \in \Omega_{x_{0}}$ is positive semi-definite.

(3) $\Omega_{x_{0}}$ is convex.

Example \%. Let the dynamics in a given location be given by $\frac{d}{d t} x=A x$ with

$$
A=\left[\begin{array}{cc}
0 & 1 \\
-2 & -3
\end{array}\right]
$$

The switching lines are given by

$$
L_{\mathrm{in}}=\lambda \underbrace{\left[\begin{array}{l}
0 \\
1
\end{array}\right]}_{a_{\mathrm{in}}} \quad L_{\mathrm{out}}=\lambda \underbrace{\left[\begin{array}{l}
1 \\
0
\end{array}\right]}_{a_{\text {out }}}
$$

As explained in Section 3.1, for a given Lyapunov function $V(x)=x^{\mathrm{T}} P x$ the gain is defined as

$$
\alpha_{P}=\frac{a_{\mathrm{out}}^{\mathrm{T}} a_{\mathrm{out}}}{a_{\text {in }}^{\mathrm{T}} a_{\text {in }}} \frac{a_{\text {in }}^{\mathrm{T}} P a_{\text {in }}}{a_{\mathrm{out}}^{\mathrm{T}} P a_{\mathrm{out}}}=\frac{p_{22}}{p_{11}}
$$

To find the optimal Lyapunov function we want to minimize $\alpha_{P}$ over $\Omega_{a_{\text {in }}}$. For this example this amounts to the minimization of $\frac{1}{p_{11}}$ or, equivalently, the maximization of $p_{11}$. Theorem 6 guarantees the optimum exists. Using Maple we found two extreme Lyapunov functions. One that minimizes $\alpha_{P}$ and one that maximizes $\alpha_{P}$ :

$$
P_{\min }=\left[\begin{array}{cc}
12.70 & 2.59 \\
2.59 & 1
\end{array}\right] \quad P_{\max }=\left[\begin{array}{cc}
.32 & .41 \\
.41 & 1
\end{array}\right]
$$

The corresponding minimum and maximum values of the gains are

$$
\alpha_{\min } \approx 0.38 \quad \alpha_{\max } \approx 2.61
$$

In Figure 2 the level curves of the two Lyapunov functions are drawn together with the phase portrait of $\frac{d}{d t} x=A x$. This clearly shows the benefit of using the non-uniqueness of Lyapunov function. The one that minimizes $\alpha$ is obviously dramatically less conservative than the one that maximizes $\alpha$. 


\section{GAIN AUTOMATA AND DETECTION OF UNSTABLE CYCLES}

Suppose we have a hybrid automaton with Hurwitz locations. If for each location that can be visited more than once the conservative gain would be $\leq 1$, then it can be seen that the hybrid automaton would be stable: since the number of locations in a trace that are visited only once (seen as a function of a trace) is bounded, there would then be a bound to the gains corresponding to the traces. However, such a condition would be unnecessarily restrictive as it would not take into account situations where a higher gain in one location can be compensated by a lower gain in another location. So we need a more sophisticated condition.

Definition 8. Let $H$ be a hybrid automaton, then an unstable cycle of $H$ is a sequence of transitions $e_{1} e_{2} \ldots e_{m}$ such that each $e_{i}$ is a transition from $l_{i}$ to $l_{i+1}$, with $l_{1}=l_{m+1}$, and $\alpha_{e_{1} e_{2}} \cdot \alpha_{e_{2} e_{3}} \cdot \ldots$. $\alpha_{e_{m} e_{1}}>1$.

Theorem 9. Let $H$ be an LCH hybrid automaton with Hurwitz locations. Then: $H$ is unstable $\Rightarrow H$ has an unstable cycle.

Theorem 9 provides us with a sufficient condition for stability, namely the absence of unstable cycles. In order to check for unstable cycles we first transform a hybrid automaton into another type of automaton.

Definition 10. A gain automaton is a tuple $G A=$ $\left(S, S^{0}, G\right)$ where

- $S$ is the set of nodes,

- $S^{0}$ is the set of initial nodes,

- $G \subseteq S \times \mathbb{R}^{+} \times S$ is the set of edges labelled with gains.

Definition 11. Let $H$ be a hybrid automaton, then the gain automaton for $H$ is defined by $G A(H)=\left(S_{H}, S_{H}^{0}, G_{H}\right)$ where

- The nodes of the gain automaton are the transitions of $H$, so $S_{H}=E$.

- The initial nodes $S_{H}^{0}$ are the transitions from an initial location of $H$.

- For each pair of transitions $e, e^{\prime}$ in $H$ such that $\stackrel{e}{\longrightarrow} l \stackrel{e^{\prime}}{\longrightarrow}$ there is an edge $e \stackrel{\alpha_{e e^{\prime}}}{\longrightarrow} e^{\prime}$ in $G_{H}$.

Example 12. Let $H$ be the hybrid automata of Example 2, then the gain automaton $G A(H)$ is given in Figure 3.

We define an algorithm on the gain automaton of a hybrid automaton for the detection of unstable cy-

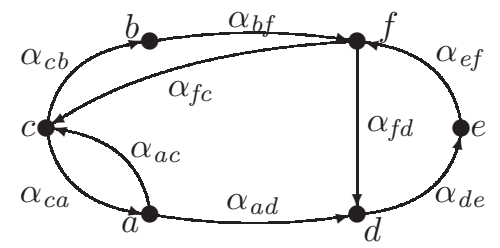

Fig. 3. Example of a gain automaton

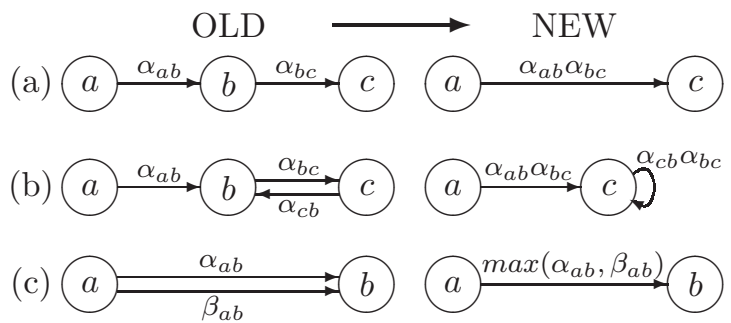

Fig. 4. Basic steps of the algorithm

cles. This algorithm is inspired by the well-known algorithm for transforming an automaton into an equivalent regular expression (see e.g.(Hopcroft et al., 2001; Linz, 2001)). It works by successively deleting nodes of the gain automaton, while transforming the edges. The basic steps of the algorithm are:

Node elimination: a node is eliminated. Each possible pair of an incoming and outgoing edge of this node leads to a new edge, labelled with the product of the gains, as illustrated in Figure $4(\mathrm{a})$.

Double edge elimination: if two edges have the same initial and final node they are transformed into a single edge, labelled with the maximum of the gains, as illustrated in Figure 4(c).

Loop edge analysis: it is possible that deleting a node creates a loop edge, as illustrated in Figure 4(b). If the gain of such a loop edge is $>1$ (we call this an unstable loop edge) the algorithm is terminated. Otherwise, the loop edge is removed.

Algorithm 1. Input: a gain automaton $G A$.

check all loop edges;

if an unstable loop edge is found

then terminate;

remove all loop edges;

repeat

eliminate a state;

eliminate all resulting double edges;

analyse all resulting loop edges;

if an unstable loop edge is found

then terminate;

remove all loop edges

until there is only one state

Theorem 13. Let $H$ be an LCH hybrid automaton with Hurwitz locations. Then: Algorithm 1 
detects an unstable loop edge in $G A(H) \Leftrightarrow H$ contains an unstable cycle.

The number of nodes in $G A(H)$ is quadratic in the number of nodes of $H$, and the complexity of Algorithm 1 is linear in the number of nodes of $G A$, so the complexity of Algorithm 1 is quadratic in the number of nodes of $H$. This means we have a computationally efficient way of checking the sufficiency condition for stability, viz. the absence of unstable cycles.

\section{CONCLUSIONS}

We have derived a sufficient condition for the stability of a hybrid automaton, viz. the absence of unstable cycles, together with an algorithm for efficiently checking this condition. In doing this we have made use of both systems theoretic concepts (in calculating the conservative gains) and computer science concepts (in checking the cycles in the gain automaton), thereby doing justice to both the continuous and the discrete aspects of hybrid systems.

The approach could in principle be adopted to non-linear dynamics in the location, provided there is a quadratic Lyapunov function. It would be interesting to see if the gain estimation could also be performed for other types of dynamics. Furthermore it would be a challenge to widen the class of hybrid systems to which this approach can be applied. Finally, if the gain estimation could also be performed for unstable locations the approach might be applied to study the stabilization of instable dynamics within the hybrid automaton framework.

\section{REFERENCES}

Agrachev, Andrei A. and Daniel Liberzon (2001). Lie-algebraic stability criteria for switched systems. SIAM J. Control Optim. 40(1), 253269.

Alur, R., C. Courcoubetis, T.A. Henzinger and P.H. Ho (1993). Hybrid automata: an algorithmic approach to the specification and verification of hybrid systems.. In: Hybrid Systems I (R.L. Grossman, A. Nerode, A.P. Ravn and H. Rischel, Eds.). Lecture Notes in Computer Science 736. Springer Verlag. pp. 209-229.

Alur, Rajeev and David L. Dill (1994). A theory of timed automata. Theoretical Computer Science 126, 183-235.

Branicky, Michael S. (1994). Stability of switched and hybrid systems. In: Proc. 3rd IEEE CDC. pp. 3498-3503.
Branicky, Michael S. (1998). Multiple Lyapunov functions and other analysis tools for switched and hybrid systems. IEEE TAC 43(4), 475-482.

DeCarlo, Raymond A., Michael S. Branicky, Stefan Pettersson and Bengt Lennartson (2000). Perspectives and results on the stability and stabilizability of hybrid systems. Proc. of the IEEE 88(7), 1069-1082.

Henzinger, Thomas A. (1996). The theory of hybrid automata. In: Proceedings LICS'96. pp. 278-292.

Hopcroft, John E., Rajeev Motwani and Jeffrey D. Ullman (2001). Introduction to Automata Theory, Languages, and Computation. 2nd edition ed.. Adisson-Wesley.

Johansson, Mikael and Anders Rantzer (1998). Computation of piecewise quadratic Lyapunov functions for hybrid systems. IEEE TAC 43(4), 555-559.

Koutsoukos, Xenofon D. and Panos J. Antsaklis (2001). Design of stabilizing switching control laws for discrete and continuous-time linear systems using piecewise-linear Lyapunov functions. ISIS Technical Report ISIS-2001002. ISIS.

Liberzon, Daniel and A. Stephen Morse (1999). Basic problems in stability and design of switched systems. IEEE Control Systems Magazine 19(5), 59-70.

Linz, Peter (2001). An Introduction to Formal Languages and Automata. 3rd edition ed.. Jones and Bartlett publishers.

Michel, Anthony N. (1999). Recent trends in the stability analysis of hybrid dynamical systems. IEEE Transactions on Circuits and Systems - I 45(1), 120-134.

Mignone, Domenico, Giancarlo Ferrari-Trecate and Manfred Morari (2000). Stability and stabilization of piecewise affine and hybrid systems: an LMI approach. In: Proc. 39th IEEE CED. pp. 504-509.

Pettersson, Stefan and Bengt Lennartson (1996). Stability and robustness for hybrid systems. In: Proc. 35th IEEE CDC. pp. 1202-1207.

Polderman, J.W. and J.C. Willems (1997). Introduction to mathematical systems theory: a behavioral approach. Vol. 26 of Texts in Applied Mathematics. Springer. New York NY, USA.

Van der Schaft, A. J. and J. M. Schumacher (2000). An Introduction to Hybrid Dynamical Systems. Vol. 251 of Lecture Notes in Control and Information Sciences. Springer Verlag. Lonon.

Ye, Hui, Anthony N. Michel and Ling Hou (1998). Stability theory for hybrid dynamical systems. IEEE TAC 43(4), 461-474. 